\title{
MicroRNA function in domestic animal physiology and diseases: a promising diagnostic tool for veterinary use ${ }^{*}$
}

\author{
KAROLINA A. CHODKOWSKA, TOMASZ SADKOWSKI, PIOTR OSTASZEWSKI*
}

\begin{abstract}
Department of Physiological Sciences, Faculty of Veterinary Medicine, Warsaw University of Life Sciences - SGGW, Nowoursynowska 159, 02-776 Warsaw, Poland
\end{abstract}

Chodkowska K. A., Sadkowski T., Ostaszewski P.

MicroRNA function in domestic animal physiology and diseases: a promising diagnostic tool for veterinary use

\section{Summary}

MicroRNAs (miRNAs) are small non-coding interfering RNA molecules capable of post-transcriptionally regulating gene expression through sequence-specific base pairing to messenger ribonucleic acid (mRNA). In recent years, hundreds of miRNAs have been identified in humans, various animals and plants. The action of miRNÄs has been examined in several biological processes, including tissue morphogenesis, development, cell proliferation and differentiation, apoptosis, immunity, metabolism, and major signaling pathways. Changes in miRNA expression have also been analyzed in the context of various pathological conditions, including different kinds of inflammation, cancer, cardiovascular diseases, etc. Thanks to these investigations, several miRNAs have been identified as potential sensitive diagnostic markers that may be important in monitoring physiological and pathological processes. In human medicine, microarray and real-time PCR-based diagnostic test panels with selected disease-specific miRNAs are increasingly used to predict disease occurrence or progression. In animals, they are mainly used to diagnose canine mammary cancers and infectious diseases, as well as to monitor reproduction. Until now, only few miRNAs of domestic animals have been studied in detail. Moreover, the silencing of selected miRNAs, successfully used in human medicine in diseases related to miRNA over-expression, is also emerging as a promising tool for veterinary medicine and animal breeding.

This review presents recent progress in miRNA biology in various domestic animals and shows the current state of knowledge concerning miRNAs and their potential role as a diagnostic factor in veterinary sciences.

Keywords: miRNA, domestic animals, physiology, cancer, breeding

\section{MicroRNA function and mode of action}

During the last decade, four types of silencingrelated small RNAs have been discovered: small interfering RNAs (siRNAs), microRNAs (miRNAs), repeat associated small interfering RNAs (rasiRNAs) and piwi-interacting RNAs (piRNAs). MicroRNAs are known as a class of endogenous, non-coding small RNA molecules (18-25 nucleotides) that are able to post-transcriptionally regulate gene expression through sequence-specific base pairing to mRNA. The first discovered miRNA, Lin-4, known to control the timing of C. elegans, was described in 1993 by Lee and colleagues. Another gene in the C. elegans heterolarval development, named let-7, was discovered 7 years after

*) This work was supported by the grant no. UMO-2011/03/B/NZ5/05697 from the National Science Centre (Poland).
Lin-4 (34). Most mammalian miRNA genes (about $80 \%$ ) have been identified in the intron region of either protein coding or non-protein coding transcripts. Only a small proportion of miRNA genes (20\%) are located in the exon region of non-coding RNAs. Interestingly, some miRNA genes could be related to either exonic or intronic miRNAs, depending on the alternative splicing pattern of the host genes (31), (47).

These small molecules play a crucial role in posttranscriptional gene expression control (47) and are involved in many gene regulation processes (13). A number of previous studies have shown that eukaryotes use miRNAs to regulate functions related to developmental timing, cell proliferation, differentiation, signaling, the programming of death (apoptosis) pathways (27), fat metabolism in flies in particular cell types (8), neuronal patterning in nematodes (30), and 
the modulation of hematopoietic lineage differentiation in mammals (15).

It is known that miRNAs can negatively regulate gene expression through recognizing completely and partially complementary sequences in target mRNAs for their cleavage or inhibition of mRNAs translation (2). In animals, target genes are recognized by miRNA through miRNA-complementary sites located in the 3'-untranslated regions (UTR), whereas plant miRNAs usually recognize one motif in the coding region of their targets (4).

All the miRNAs that have been identified in plants and animals (published miRNA sequences and annotation) are registered in a special database, miRBase, which has been annotated from the microRNA registry resource set up by Griffiths-Jones in 2003 (22). The data is freely available through the web interface at http://www.mirbase.org/and in a flat file form from $\mathrm{ftp}: / /$ mirbase.org/pub/mirbase).

\section{Biogenesis}

Transcription and maturation. The current models for miRNA biogenesis have shown that a compartmentalized stepwise processing of miRNAs occurs first in the nucleus and then in the cytoplasm (47). The primary transcripts of miRNA (pri-miRNA) are processed in the nucleus by the RNase III. The intermediated molecule called pre-miRNA is transported to the cytoplasm for cleavage by Dicer, which is essential for miRNA biogenesis, because organisms lacking the enzyme cannot synthesize miRNAs $(34,43)$ (Fig. 1). The two enzymes, which are candidate RNA polymerases for the pri-miRNA role in generating siRNA transcription, are pol II and pol III (4). Pol II is involved in the production of mRNAs, some non-coding RNAs (including the small nucleolar RNAs (snoRNAs) and four of the small nuclear RNAs (snRNAs) of the spliceosome). The other enzyme, pol III, produces some of the shorter non-coding RNAs (e.g. tRNAs, 5S ribosomal RNA, and the U6 snRNA).

Maturation of the mammalian miRNA, RICS assembly. The microRNA maturation process consists of several stages. The first stage is related to the nuclear cleavage of the pri-miRNA. At this stage, a 60-70 nt stem loop intermediate miRNA precursor (pre-miRNA) is liberated with Drosha RNase III endonuclease engagement $(35,68)$. In the nucleus, it cleaves the RNA duplex with a staggered cut typical of this kind of enzyme, and thus the base of the pre-miRNA stem loop has a 5_phosphate and _2 nt 3_overhang $(4,5$, $34)$. During the next stage, pre-mi $\bar{R} N A$ is actively transported from the nucleus to the cytoplasm by Ran-GTP and the export receptor Exportin-5 $(39,66)$. The cut made by Drosha in the nucleus defines one end of the mature miRNA, while the other one is related to Dicer activity in the cytoplasm. After being exported from the nucleus into the cytoplasm and undergoing cleavage processes, imperfect matched miRNA/

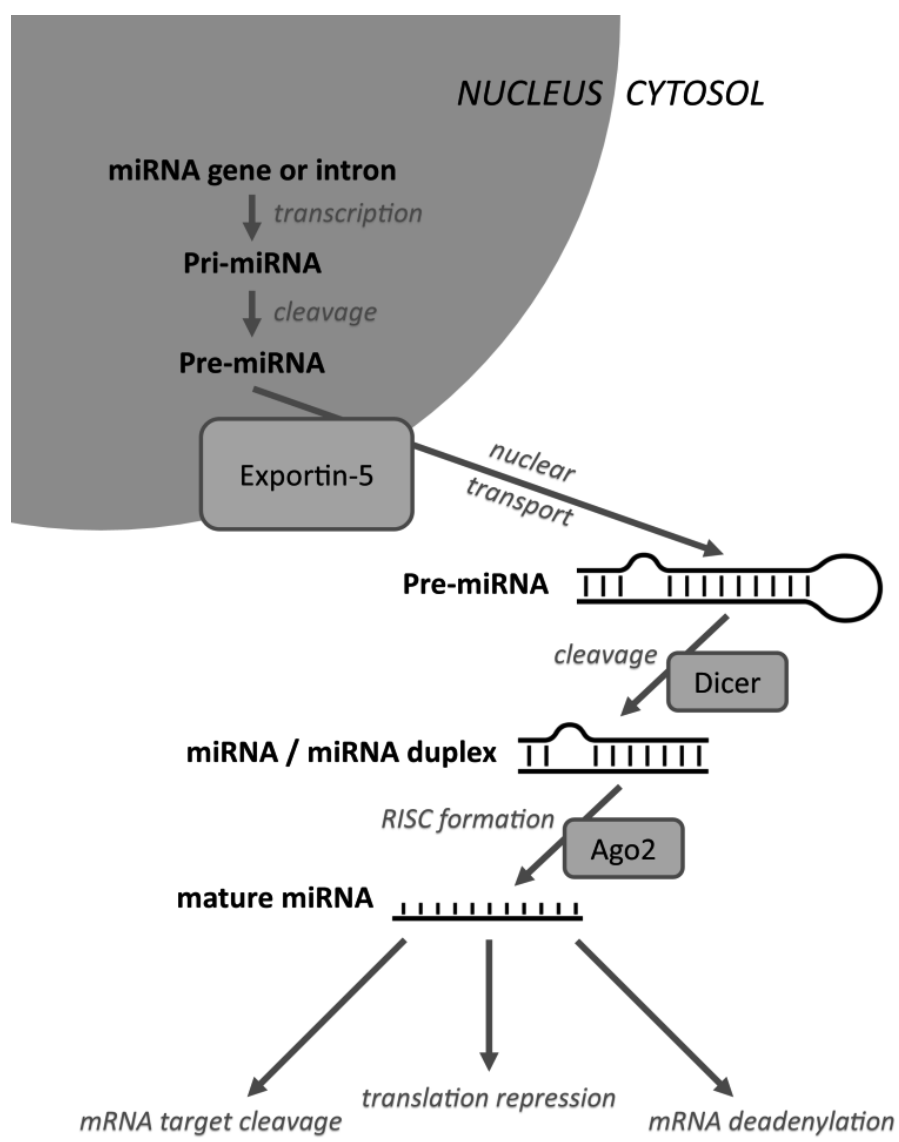

Fig. 1. Biogenesis of animal miRNA

miRNA duplexes are loaded into the Argonaute (Argo) protein to generate the RNA-induced silencing complex (RISC) (40). The mature miRNA which is loaded into RISC (it identifies target messages on the basis of perfect or nearly perfect complementarity between the siRNA and the mRNA) interacts with the 3' UTR. In RISC, miRNAs can mediate the down-regulation of gene activity in two modes: translational inhibition or targetted mRNA cleavage. The degree of complementarity between the miRNA and the target gene in combination with an Argonaute family protein is the criterion of the mode choice (64). Near-perfect complementarity results in cleavage, whereas partial complementarity leads to translational inhibition. The exact mechanism of the latter process is still unknown, but there are a few theories on translational inhibition. One of them describes the possibility of miRNA-targeted mRNA being sequestered on polysomes (42). Another probable theory is that miRNA-targeted miRNAs could be recruited to P-bodies, where they are depleted of the translation machinery and eventually degraded $(38,50)$ (Fig. 1).

MicroRNA expression. Tests for the analysis of miRNA expression involve the use of microarrays, Northern blots, in situ hybridization, and solution hybridization or a proprietary quantitative Real Time Reverse Transcriptase-Polymerase Chain Reaction (qRT-PCR) platform. However, the method most commonly used to determine miRNA expression is 
the microarray technology. It is a very powerful highthroughput tool that can be used to monitor the expression of thousands of miRNAs at once in a single experiment. A typical microarray platform consists of a few steps: the purification of mature miRNAs from cells or tissues, the separation of small RNA and large RNA $(>200 \mathrm{nt}$ ), the enrichment of miRNAs and labelling; the design of the microarray (including probe design, control design), probe spotting and array hybridization; and finally, the analysis of results through measurement, quantification, normalization, and validation. It is also possible to use a special commercial array prepared for selected animal species (67).

Another commercially used method for miRNA expression analysis is qRT-PCR, which is considered to be a gold standard because of its unparalleled sensitivity and specificity. There are several commercially available miRNA RT-qPCR methods. Among them, the Taqman assays use a miRNA-specific stem-loop reverse transcription primer to generate cDNA for subsequent hydrolysis probe qPCR amplification. Other assay (the miRCury LNA Universal RT PCR) involves the poly(A)tailing of mature miRNAs and the subsequent use of a poly $(\mathrm{T})$ reverse transcription primer containing a $3^{\prime}$ degenerate anchor and a 5' universal tag to generate cDNA for amplification by SYBR Green qPCR using miRNA-specific forward and reverse primers containing locked nucleic acids (LNAs). The primers are commercially available or easy to design on the basis of the sequence identified by the microarray (46).

MicroRNA in animal physiological function and diseases. Although miRNAs were recognized as key regulators of mammalian genome only a few years ago, there have already appeared a stunning number of publications documenting the physiological and pathological roles of miRNA in animal diseases (Tab. 1).

There are four probable general theories about the ways in which miRNA-mRNA interactions could be disrupted and cause disease. However, not all of them have been observed as yet (43).

(1) A microRNA may acquire a mutation and, as a result, lose its function. Although there is some degree of redundancy among miRNAs, it occurs at a gross level. A subtle disease may result from the loss of even a single miRNA gene.

(2) A theory on the possible gain-of-function mutation in a miRNA. He et al. (25) describe it as over-

Tab. 1. Selected miRNAs and their function in different animal species

\begin{tabular}{|c|c|c|}
\hline miRNA & Process and function & References \\
\hline \multicolumn{3}{|l|}{ Canis domesticus } \\
\hline miR-29b, miR-21 & Canine mammary tumors/up-regulated in malignant tumor & (7) \\
\hline miR-15a, miR-16 & Canine tumors/down-regulated in canine ductal carcinomas & (7) \\
\hline miRs-181b, $-21,-29 b$,and let-7f & Canine tumors/up-regulated in canine tubular papillary carcinomas & (7) \\
\hline miR-494 & Canine tumors/highly expressed in Rottweiler OSA & (18) \\
\hline miR-30b, miR-133b & Biomarkers of ACVIM & (26) \\
\hline miR-34a, miR-16, miR-103b, and miR-106b & Canine tumors/up-regulated in transitional cell carcinoma & (64) \\
\hline \multicolumn{3}{|l|}{ Felis domesticus } \\
\hline miR-122 and miR-139b & Diabetes & (19) \\
\hline $\begin{array}{l}\text { miR-381-3p, miR-486-3p, miR-4751, miR-476c-3p, miR-5700, } \\
\text { miR-513a-3p, and miR-320e }\end{array}$ & Up-regulated in hypertrophic cardiomyopathy & (61) \\
\hline \multicolumn{3}{|l|}{ Equus caballus } \\
\hline miR-21, $-145,-224,-378,-132$ and -212 & $\begin{array}{l}\text { Follicule development/the regulation of cell survival, steroidogenesis and } \\
\text { differentiation during follicle selection and ovulation in the monovular ovary }\end{array}$ & (49) \\
\hline miR-21, $-23 a,-145,-503,-224,-383,-378,-132$, and -212 & Follicle maturation & (16) \\
\hline miR-181A, miR-375, and miR-513A-3p & $\begin{array}{l}\text { Cell-secreted vesicles miRNAs/differences in miRNAs expression in old vs. } \\
\text { young mares }\end{array}$ & (52) \\
\hline miR-1, -133, -23a, $-30 b,-195$, and -339 & Higher expression in RER TB & (3) \\
\hline miR-195 & Higher expression in PSSM-Cob & (3) \\
\hline miR-140 & Chondrogenesis & (9) \\
\hline \multicolumn{3}{|l|}{ Bos taurus } \\
\hline miR-125b, -141, -181a, -199b, -484 and -500 & Lactogenesis/Mastitis & $(36,37)$ \\
\hline hsa-aga-3155, $-8197,-6727,-11796,-14189,-6125,-13659$ & Spermatogenesis & (20) \\
\hline miR-221 and miR-222, miR-126, miR-145, and miR-335 & Chondrogenesis & (17) \\
\hline \multicolumn{3}{|l|}{ Animal infectious disease } \\
\hline gga-miR-15b & Marek's disease in chicken & $(56)$ \\
\hline miR-181 & Positive regulation of immune response/PRRSV & (24) \\
\hline
\end{tabular}


expression by the amplification of the miRNA locus, which may work similarly to the over-expression of an oncogene, resulting in cancer.

(3) In a third theory, a programmed target site may acquire a mutation. It may result in the target no longer being able to bind to the miRNA and releasing the gene from regulation by that miRNA.

(4) The last theory is related to the fact that a gene may acquire a new and undesired miRNA target sequence that results in its aberrant silencing. There are many sequences that are only one mutation away from becoming a target for one of numerous miRNAs that may be expressed in the same tissue. Some of these mutations may cause an undesired reduction in gene activity and may cause disease (43). However, in a study conducted by Abelson et al. (1), it was shown that the acquisition of a miRNA target site in the gene encoding the axon guidance molecule SLITRK1 is associated with the development of Tourette's syndrome, a neurological disorder that affects motor function.

A growing number of data suggest that miRNAs can be utilized as potential biomarkers not only for the diagnosis, but also for the prognosis of a variety of diseases, such as human and animal cancer, orthopaedic disorders, cardiovascular disease, some infectious diseases, etc. Some of miRNAs can be utilized not only for the monitoring of therapy, but also for the external regulation of miRNA expression (e.g. silencing) and may soon become effective treatment methods for various health problems in animals.

MicroRNA expression in animal cancer. Cancer is a complex genetic disease involving structural and expression abnormalities of both coding and non-coding genes. MicroRNAs play an important role in differentiation, proliferation, and tumorgenesis by binding to mRNAs, thus inhibiting translation. The causes of the widespread differential expression of miRNA genes in malignant cells, compared with normal, healthy cells, can be related to the location of these genes in cancerassociated genomic regions by epigenetic mechanisms and by alterations in the miRNA processing machinery (11). The microRNA expression profiling of different tumors in domestic animals has identified signatures associated with their diagnosis, staging, progression, prognosis, and response to treatment.

As in humans, cancer is the leading cause of death in older domestic and wild animals. Thanks to the development of early diagnostics and therapy, the life comfort of these animals is better than it was years ago. However, scientists are still searching for new tools that could be used for detecting cancer in its earliest stage or even before symptoms arise. The analysis of miRNA can be one of these tools. There are a number of studies on the comparison of normal and malignant cells in different animals.

Boggs et al. (7) have compared malignant canine mammary tumors with normal canine mammary tissue. The results showed that miR-29b and miR-21 have a statistically significant up-regulation in cancerous samples. Another observation was that ten canine miRNAs follow the same pattern of expression as in humans, except for miR-145, which does not show differences in expression between normal and cancerous canine samples. Furthermore, the authors discovered that miR-15a and miR-16 show a significant down-regulation in canine ductal carcinomas, while miR-181b, $-21,-29 b$, and let-7f show a significant up-regulation in canine tubular papillary carcinomas.

Another interesting study related to miRNA in animal cancers has been presented by Uhl et al. (58). In this study, human cancer microRNA qPCR arrays were used to establish the miRNA profiles of 4 canine lymphoid cell lines: 2 of B-cell origin and 2 of T-cell origin.

The results showed that over $90 \%$ of human miRNA probes detected canine miRNAs. The microRNA analysis of canine lymphoid cell lines identified 12-15 miRNAs that were either 10 -fold over-expressed or 10-fold under-expressed compared to normal canine peripheral blood mononuclear cells. Furthermore, the authors observed that the relative expression of 6 miRNAs known to be up-regulated in human chronic lymphoid leukemia was determined in four canine lymphoid cell lines, and the expression of miR-20a and miR-92 were markedly increased in these cell lines. The results of this study show that miRNA profiles have a potential predictive, diagnostic, and prognostic potential in canine lymphoma.

The same authors began generating miRNA profiles of spontaneous canine B- and T-cell tumors, using cancer microRNA qPCR arrays developed for humans. This analysis showed that at least $20 \%$ of miRNAs were up-regulated in tumors relative to PBMC (peripheral blood mononuclear cell). Nineteen microRNAs were increased and one decreased in T-cell tumors, nine were increased in B-cell tumors, and eight were up-regulated in both B- and T-cell tumors (60). Another observation was that the magnitude of miRNA upregulation was generally greater in T-cell tumors than in B-cell tumors. It is one more study which indicates that groups of miRNAs can be used as diagnostic markers in canine lymphoma.

In different studies, Uhl et al. (59) determined that miRNA expression in canine tumors could be assessed using a commercially available human cancer miRNA qPCR array. MicroRNA expression in six different canine lymphoid cell lines and in naturally occurring canine B- and T-cell lymphomas was compared using RNA harvested from normal canine peripheral blood mononuclear cells and normal lymph nodes (LN) as controls. The authors observed increases in miRNAs known to up-regulate oncogenes (miR-19a+b, miR-17-5p) and a decreased expression of miRNAs with tumor suppressor functions (miR-203, miR-218, and miR-181a), also seen in human lymphoid malignancies. Their findings of miRNA dysregulation in 
canine lymphoid cell lines and clinical cases of lymphoma emphasize the potential of canine lymphoma as an important spontaneous, large-animal model of human B- and T-cell lymphomas.

Another type of animal cancer, canine osteosarcoma (OSA), which is the most common bone tumor in dogs, was the subject of a detailed analysis of miRNA expression (18). This study was prompted by the fact that some breeds, such as Rottweilers and Greyhounds, are at a higher risk of developing OSA, which may suggest that heritable factors play a role in this disease. RNA was isolated from a panel of seven normal canine tissues and 48 primary canine OSA tumors from Greyhound, Golden Retriever, Rottweiler, and mixed breed dogs. The results showed that 189 miRNAs were differentially expressed in Greyhound, Rottweiler, Golden Retriever, and mixed breed dog tumors. In an expanded cohort of Greyhound and Rottweiler tumors, miR-494 was highly expressed in Rottweiler OSA as compared to Greyhound OSA or normal canine osteoblasts from various dog breeds, including one Rottweiler. The characterization of miRNA expression in canine osteosarcoma will probably help better understand the biology of the disease and has the potential to identify diagnostic/prognostic factors and targets for therapeutic intervention.

MiRNA in animal reproduction and breeding. Post-transcriptional gene regulation plays a vital role in male and female germ cell function. However, this regulatory process in somatic cells and its impact on reproductive tissue development and function are still not well understood. To understand the role that miRNA-mediated post-transcriptional gene regulation plays in female and male reproduction, several animal models have been studied.

Cameron et al. (12) observed exosomes containing miRNA as an indicator of pregnancy status in mares. The authors evaluated serum expression levels of 380 miRNAs collected from pregnant and non-pregnant mares on the 16th day after ovulation. They found seven specific miRNAs differentially expressed in pregnant compared with non-pregnant mares. Two miRNAs were expressed in pregnant mares only (miR-146b-5p, miR-424), whereas two other miRNAs were present in non-pregnant mares only (miR-26b, miR-183). These findings suggest that miRNAs, most likely released from the endometrium or the embryo, enter the mare's circulation via exosomes. The authors suggest that since pregnancy status was the primary difference between samples collected from the same mare, the differentially expressed miRNAs from exosomes must be associated with pregnancy. Finally, they conclude that the differential expression of 7 miRNAs (miR-130b, miR-140-3p, miR-195, miR-433, miR-508-3p, miR-767and miR-770) at day 16 post ovulation can be used for pregnancy determination in mares.
Relatively little is known about the physiological roles of miRNAs during follicular development. Only a few studies have been conducted on this subject. Schauer et al. (49) have presented study of the involvement of miRNAs in equine follicle development. The authors determined changes in the levels of miRNAs in relation to follicle selection, maturation, and ovulation in the monovular equine ovary. Follicular fluid was aspirated during ovulatory cycles from the dominant (DO) and largest subordinate (S) follicles of an ovulatory wave and the dominant (DA) follicle of a mid-cycle anovulatory wave. The results showed that, compared with DO and DA follicles, $\mathrm{S}$ follicles had higher follicular fluid levels of miR-145 and miR-378. The second part of the experiment dealt with follicular fluid and granulosa aspiration from dominant follicles before (DO) and $24 \mathrm{~h}$ after (L) the administration of an ovulatory dose of hCG. The results showed that the levels of miR-21, miR-132, miR-212, and miR-224 were increased in L follicles; this was associated with a reduced expression of the putative miRNA targets, PTEN, RASA1, and SMAD4. These results may suggest that there is a physiological involvement of miR-21, miR-145, miR-224, miR-378, miR-132, and miR-212 in the regulation of cell survival, steroidogenesis, and differentiation during follicle selection and ovulation in the monovular ovary.

A similar study has been performed by Donadeux et al. (16). The researchers compared the miRNA expression of equine ovulatory and anovulatory follicles. They gained insight into the involvement of a subset of miRNAs, including miR-21, miR-23a, miR-145, miR-503, miR-224, miR-383, miR-378, miR-132, and miR-212, during follicle maturation. In their experiment, follicular fluid was aspirated from dominant follicles during the ovulatory season (from July to October) and the anovulatory season (from January to March) in each of 5 mares, and the levels of steroids, IGF1, and miRNAs were analyzed by immunoassays and quantitative PCR. The results showed that, compared with ovulatory follicles, anovulatory follicles had higher mean levels of miR-21, miR-23b, miR-378, and miR-202 and tended to have higher $(\mathrm{P}=0.06)$ levels of miR-145. The levels of miR-224 and miR-383 could not be detected in follicular fluid. This observation may indicate a physiological association between increases in follicular miRNA levels and seasonal anovulation in mares. Further studies should elucidate the precise involvement of miR-21, miR-23b, miR-145, miR-378, and miR-202 in follicle maturation in the mare.

MicroRNAs related to ovarian follicles have also been analyzed by da Silveira et al. (52). The goals of their study were 1) to identify cell-secreted vesicles containing miRNAs and proteins within ovarian follicular fluid and 2) to determine whether the miRNA level differs in exosomes isolated from follicular fluid in young compared with old mares. The authors 
demonstrated the presence of vesicles resembling microvesicles and exosomes in ovarian follicular fluid. Moreover, proteomic analysis showed that follicular fluid-isolated exosomes contained both known exosomal proteins and proteins not previously reported in isolated exosomes. The results showed that miRNAs in microvesicle and exosome preparations isolated from follicular fluid were also present within surrounding granulosa and cumulus cells. These observations may suggest that cell communication within the mammalian ovarian follicle may involve the transfer of bioactive material by microvesicles and exosomes. MiRNAs present in exosomes from ovarian follicular fluid varied with the age of the mares, and different miRNAs (14) were detected in follicular fluid from young vs. old mares. Three miRNAs (miR-181A, miR-375 and miR-513A-3p) were expressed in significantly $(P \leq 0.05)$ greater amounts in exosomes isolated from the follicular fluid of old mares compared with young mares.

Also the miRNA profile of other species has been analyzed in studies related to reproduction. Tesfaye et al. (55) identified and described the expression profiling of miRNAs during bovine oocyte maturation using a heterologous approach. The authors observed the differential expression of 59 miRNAs, 31 and 28 of which were preferentially expressed in immature and matured oocytes, respectively. They also reported the identification of 32 orthologous miRNAs by a heterologous approach. Tesfaye et al. also found that the expression profiling of selected miRNAs during the pre-implantation stage embryos showed a distinct temporal expression pattern.

In the mammary gland, most of the miRNAs studied have functions related to immunity. This is related to their role in regulating the development of immune cells and in modulating innate and adaptive immune responses. Li et al. (37) identified novel and differentially expressed miRNAs in peripheral blood from healthy and mastitis-affected Holstein cattle by Solexa sequencing and bioinformatics. The authors detected 608 precursor hairpins (pre-miRNAs) encoding for 753 mature miRNAs and identified 173 unique miRNAs (out of $753,22.98 \%$ ) that had significant differential expression between healthy and mastitis-affected Holstein cattle $(\mathrm{P}<0.001)$. What was interesting was that most differentially expressed miRNAs (118 out of $173,68.21 \%$ ) belonged to the chemokine signaling pathway involved in immune responses. MiRNA profile changes in mastitis-affected cows may be important information and, as the authors suggest, may play a crucial role in the treatment of mastitis in Holstein cattle. Bovine miRNA has also been analyzed in studies by Li et al. (36). The authors described the expression profile of microRNAs from lactating and non-lactating bovine mammary glands (MG) and the identification of miRNAs related to lactation. The results showed a total of 56 miRNAs in lactating MG with significant differences in expression when compared to non-lactating
MG. Integrative miRNA target prediction and network analysis approaches were employed to construct an interaction network of lactation-related miRNAs and their putative targets. Using a cell-based model, six miRNAs (miR-125b, miR-141, miR-181a, miR-199b, miR-484, and miR-500) were studied to reveal their possible biological significance. This investigation was an important step towards understanding the mechanisms of lactogenesis.

A similar study has been presented by Ji et al. (29). The authors identified novel and differentially expressed microRNAs of dairy goat mammary gland tissues. They obtained 19,044,002 and 7,385,833 clean reads and identified 1,113 conserved known microRNAs, as well as 31 potential novel microRNA candidates. A total of 697 conserved microRNAs were significantly differentially expressed, 272 microRNAs were up-regulated, and 425 microRNAs were downregulated during peak lactation. This was the first study in which the global expression of miRNA in Capra hircus was analyzed and proved helpful in the study of mammary gland physiology and lactation. Another interesting study has been presented by Castro et al. (14), who analyzed the microRNA expression profiling of elongated cloned and in-vitro-fertilized bovine embryos. The authors identified miRNAs expressed in bovine embryos on the $17^{\text {th }}$ day of development (day $0=$ day of nucleus transfer or in vitro fertilization) during elongation. They observed 39 miRNAs expressed in elongated day-17 cloned bovine embryos produced by HMC, 32 miRNAs expressed in timematched IVP embryos, and 22 miRNAs in somatic cells used for cloning. These results could have profound implications for understanding nuclear reprogramming in somatic cloning, as well as the role of miRNAs in early mammalian pre-implantation development.

Not only female reproductive functions have been analyzed on the basis of miRNA. Govindarayu et al. observed the dynamics of microRNAs in bull spermatozoa (20). They found that an abundance of microRNAs were present in bovine spermatozoa, but only seven were differentially expressed: hsa-aga-3155, -8197, $-6727,-11796,-14189,-6125$, and 13659 . The abundance of miRNAs in spermatozoa and the differential expression in sperm from high- vs. low-fertility bulls suggest that miRNAs possibly play important functions in the regulating mechanisms of bovine spermatozoa, which can help improve the understanding of mammalian gametogenesis and early development.

All these studies show that miRNA expression analysis not only can be helpful in diagnosing some irregularities (early embrionic death, mastitis, infertility, etc.) in both male and female reproduction, but can also be used as a monitoring tool in breeding (estrus cycle, semen quality, etc.).

It is worth mentioning that there are a number of studies concering the transcriptomic analysis of the miRNA profile according to the breeding type. This 
applies in particular to pigs as well as beef cattle (different miRNA expression in muscle tissue in different breeds) and dairy cattle (miRNAs in muscle, mammary gland) $(51,62)$. All these studies indicate that the high developmental potential of the muscular tissue and mammary gland in dairy/meat cattle, leading to high milk/meat productivity, also depends on a specific miRNA expression pattern.

MiRNAs related to cardiac, skeletal muscle and cartilage physiology and pathology. There are number of studies dealing with miRNAs related to muscle development. Chen et al. (15) has found that miR-1, especially in cell culture, promotes myogenesis by targeting histone deacetylase 4 (HDAC4), a transcriptional repressor of muscle differentiation. On the other hand, HDAC4 is also a possible target of miR-140 during osteoblast differentiation and skeletogenesis, showing that two different miRNAs can target the same gene during differentiation (57). MiR-1 is located in a genomic cluster with miR-133. However, these two miRNAs differ in their seed sequence and have distinct functions (15). In contrast to miR-1, miR-133 may inhibit muscle differentiation and promotes proliferation, which is related to serum response factor repression.

Another miRNA, miR-181 is strongly up-regulated during myoblast differentiation and inhibits the expression of Hox-A11, which is a repressor of differentiation (41). MiR-181, which is hardly detected in muscles, might also be involved in establishing a muscle phenotype, while miR-1 and miR-133 are involved in muscle maintenance $(32,41)$. What is interesting, miR-181 can also induce the differentiation of hematopoietic stem cells to B-lineage cells, which suggests its dual role in different cell types (15).

Interesting studies of the role of miRNA in animal muscle during certain diseases have been performed by Barrey et al. (3). The objective of their research was to identify miRNA candidates in the muscles of control and affected horses suffering from polysaccharide storage myopathy (PSSM) and recurrent exertional rhabdomyolysis (RER). Muscle and blood samples were collected from horses allocated into 4 groups: French Trotters (3 control-FT vs. 3 RER-FT) and Norman Cob (5 control-Cob vs. 9 PSSM-Cob). The results showed that all the miRNA candidates were significantly detected in muscles and some in blood samples. Variance analysis revealed highly significant effects of the miRNA type, breed, and pathology on miRNA expression. A higher expression of mir-1, -133 , $-23 \mathrm{a},-30 \mathrm{~b},-195$, and -339 was detected in RER-FT vs. control-FT, and a higher expression of mir-195 was found in PSSM-Cob vs. control-Cob. The authors also observed that the miRNA profile was different between breeds for miR-181, -188 , and -206 . The miR-1, -133 , $-181,-195$, and -206 were detected in the blood of control-Cob and PSSM-Cob horses. It was the first study of the muscular miRNA profile in equine myopa- thies. Interestingly, the RER miRNA profile was more specific and contrasted than the PSSM one.

Not only the skeletal muscle miRNA profile in various physiological and pathological states has been analyzed by scientists. Steudemann et al. (53) screened the miRNA expression profile of canine serum with miRNA microarray and compared expression patterns for a group of Doberman Pinschers with dilated cardiomyopathy (DCM) and healthy controls. The results showed that numerous miRNAs can be detected in canine serum. Differences in miRNA expression could be detected between healthy and DCM dogs, but the results did not reach statistical significance, most probably because of the small group size. The authors suggest that miRNAs are potential new circulating biomarkers in veterinary medicine and should be investigated in larger patient groups and other canine diseases. A similar study has been presented by Hulanicka et al. (26). The authors showed that plasma microRNAs may be potential biomarkers of chronic degenerative valvular disease in Dachshunds. In their research, they analyzed the expression of 9 miRNAs described in the literature as being involved in cardiovascular pathology in the plasma of dogs suffering from endocardiosis. The authors suggest that miR-30b could be a potential biomarker of ACVIM stage B heart failure in Dachshunds with endocardiosis, and miR-133b could be a potential biomarker of ACVIM stage C. Moreover, Weber et al. (61) analyzed serum microRNA profiles in cats with hypertrophic cardiomyopathy (HCM). The authors compared miRNA profiles in healthy cats and cats with stable congestive heart failure caused by HCM, using miRNA arrays. They detected higher expression of miR-381-3p, miR-486-3p, miR-4751, miR-476c-3p, miR-5700, miR-513a-3p, and miR-320e in the HCM group. What is interesting, the cats with primary HCM showed a distinct miRNA profile including miRNAs that had already been shown to be differentially regulated in human patients and rodent models for cardiac disease. This suggests that miRNA may be a potential marker of HBM in domestic animals.

Another interesting miRNA, miR-140, was the subject of a study presented by Buechli et al. (9). The authors investigated the expression of miR-140 during the chondrogenic differentiation of equine cord-bloodderived mesenchymal stromal cells (eCB-MSCs). The results showed that miR-140 was highly expressed in normal equine articular cartilage and that eCB-MSCs expressed significantly higher levels of this miRNA after 14 days of chondrogenic differentiation. Another finding was that miR-140 expression closely paralleled that of the cartilage-specific transcription factor Sox9, suggesting that miR-140 may be under the transcriptional regulation of Sox9 in these cells. Buechli et al. also determined the expression patterns of miR-140 targets - the chemokine (CXC motif) ligand 12 (CXCL12), a disintegrin and metalloproteinase 
with thrombosponin motifs (ADAMTS-5), and insulin growth factor binding protein 5 (IGFBP5). However, only CXCL 12 and ADAMTS-5 were repressed, while the expression of miR-140 was up-regulated. All these results may suggest that miR-140 is an important regulator of cartilage development and homeostasis in eCB-MSCs that may act, in part, through the regulation of CXCL12 and ADAMTS-5. Also bovine cartilage has been studied for the expression of miRNAs. Dunn et al. (17) analyzed the hypothesis that miRNA expression profiles in different bovine articular cartilage zones, as well as between regions subjected to different levels of weight-bearing stresses, are unique. The authors identified miRNA-221 and miR-222 as part of a subset of differentially expressed miRNA that was up-regulated in articular cartilage in the anterior, medial femoral condyle (M1), greater weight-bearing location. Another observation was that miR-126, miR-145, and miR-335 were down-regulated in monolayers of tissue-cultured chondrocytes as compared with levels determined directly from intact native cartilage. These results may suggest that miR-222 is a potential regulator of an articular cartilage mechanotransduction pathway. This study implicates miRNA in the maintenance of articular cartilage homeostasis, and therefore miRNAs are targets for articular cartilage tissue engineering and regenerative medicine.

MiRNA in infectious diseases and other noninfectious animal disorders. Apart from multicellular eukaryotes, many miRNAs have been found in viruses (23). Thanks to their small size, miRNAs are attractive as effector molecules during viral infection. Experimental evidence for the existence of viral miRNAs is based on several studies that detected miRNAs in herpes viruses and polyoma viruses $(10,21$, 23). Last year, some infectious diseases dangerous for both animals and humans were analyzed by searching for changes in miRNA in infected tissue. Zhao et al. (68) described changes in miRNA expression induced by rabies virus (RABV) infection in the mouse brain. The authors found that ten miRNAs, miR-1894-5p, miR-290-3p, miR-1901, miR-207, miR-1896, miR-715, miR-3470b, miR-146b*, miR-203, and miR-770-5p, were significantly up-regulated, and six miRNAs, miR-200a, miR-200b, miR-200c, miR-182, miR-183, and $\mathrm{miR}-429$, were significantly down-regulated upon RABV infection. They also observed that several targets of differentially expressed miRNAs were involved in the chemokine signaling pathway. These included CXCL10, a target of miR-183. MicroRNA profiles obtained from this study might help elucidate the regulatory mechanisms that mediate the host response to RABV exposure. A similar study was presented by Tian et al. (56). The authors described miRNA expression signatures induced by Marek's disease virus (MDV) infection in chickens. The results showed that chicken miRNA gga-miR-15b, which was reduced in infected susceptible chickens and splenic tumors, controlled the expression of ATF2 (activating transcription factor 2). ATF2 was significantly increased in the same group. This indicated that the differential expression of miRNA in resistant and susceptible chickens was caused by MDV infection, which effectively influenced ATF2 protein expression. The latter finding may prove important in predicting resistance or susceptibility to Marek's disease.

Another animal virus, bovine herpesvirus, has been analyzed in detail by Tang et al. (54). The authors compared bovine herpesvirus 5 (BoHV-5) with herpesvirus 1 (BoHV-1). They identified 11 miRNA genes encoded by BoHV-5 genome that were processed into 16 detectable mature miRNAs in productive infection as determined by deep sequencing. The authors also found that 6 out of 16 miRNA genes were present as two copies in internal repeat and terminal repeat regions, resulting in a total of 17 miRNA encoding loci distributed in both DNA strands. They also observed that BoHV-5 shared only one conservative miRNA with BoHV-1, which was located at the upstream of oriS. These data suggest that BoHV-5 encodes a different pattern of miRNAs, which may regulate the life cycle of BoHV-5 and might account for its different pathogenesis compared with BoHV-1. MiR-181, involved in the positive regulation of immune response, was the subject of a study that analyzed porcine reproductive and respiratory syndrome virus (PRRSV) infection (24). The authors showed that the direct impairment or even inhibition of this disease was related to miR-181 expression. Previous animal experiments provided direct evidence that miR-181 could repress PRRSV replication in vivo. In this study, they also demonstrated that therapeutic miR-181 administration could relieve symptoms in pigs infected with the highly pathogenic PRRSV strain and that treated pigs survived longer.

Another study of miRNA profiling in pigs concerned Actinobacillus pleuropneumoniea and pseudorabies. The authors identified some miRNAs (miR-664-5p, miR-451 and miR-15a) that may play important roles in the immune and inflammatory response to both pathogens $(44,45)$.

Vinall et al. (64) determined the expression of microRNA (miRNA) in urinary bladder samples from dogs with grossly normal urinary bladders, inflammatory bladder disease, and transitional cell carcinoma (TCC), as well as in cells of established canine TCC cell lines. The authors observed that the expression of miR-34a and miR-106b was significantly higher in TCC samples than it was in samples of grossly normal bladders. They also found that the expression of miR-34a, miR-16, miR-103b, and miR-106b was higher in TCC samples than it was in bladder samples from dogs with inflammatory bladder disease. Vinall et al. suggest that this finding may have clinical relevance because currently available diagnostic tests cannot be used to differentiate these tissues, and inflammatory bladder disease and TCC are both prevalent in dogs. 
There are only a few studies specifically devoted to the use of miRNAs in clinical veterinary medicine $(19,28,53)$. Most of them focus on the potential diagnostic role of miRNAs in early detection of some diseases or describe novel miRNA profiling in particular species/breeds or types of disease. Current studies mostly compare miRNA profiles of healthy and pathologically changed organisms.

\section{Conclusions}

Over the last decade, many studies have dealt with the role of microRNA in animal development, physiological function, and disease. Most miRNA studies, however, are conducted on laboratory animals and cell lines with the primary objective of understanding the role of miRNAs in human development and diseases (i.e. using animal

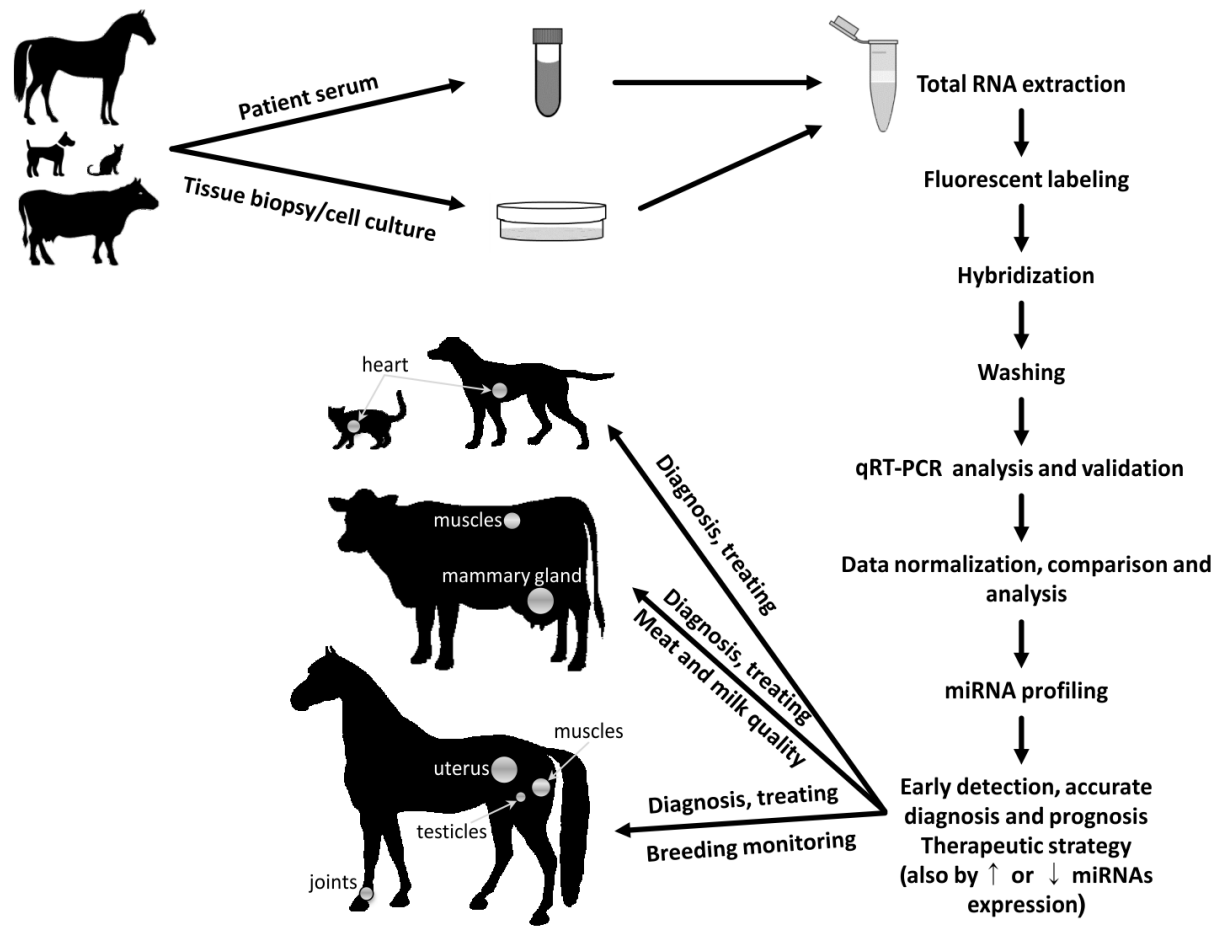

Fig. 2. MiRNA, a potential diagnostic tool for veterinary medicine and animal science. Basic miRNA analysis protocol models for human medicine). Although organ- and breed/species-specific miRNA signatures have been documented in various domestic and non-domestic animals, studies that have directly addressed diseases or physiological dysfunctions specific to companion, avian, and exotic animals are extremely limited (6).

In this review, we have presented numerous examples suggesting that some physiological processes and diseases in domestic animals may be caused by a dysfunction of miRNAs. MicroRNA is not only a scientific tool in genetics. Another use of miRNA in animal science and veterinary medicine is related to drug response. However, there are still few studies on the direct use of miRNAs and miRNA-related mechanisms in the development of therapeutic strategies.

A growing number of papers dealing with the assessment of the miRNA profile in different species of domestic animals show that, as in human medicine, miRNA profiling may become a useful diagnostic tool in veterinary practice. A large group of miRNAs have already been validated, and variation in their expression is used to monitor changes in physiology (e.g. reproduction) and as an additional diagnostic method in oncology and non-infectious and infectious diseases. The promising findings of these studies and the growing interest in the analysis of miRNA profiles in animals of different health status may eventually lead to the wide use of this method in various branches of veterinary medicine (as potential biomarkers for diagnosing, subtyping, and estimating the progression of many diseases) and its greater availability in clinical practice (as the assessment of the transcriptomic profile; cDNA microarrays and RT method PCR) (Fig. 2).

\section{References}

1. Abelson J. F., Kwan K. Y., O'Roak B. J., Baek D. Y., Stillman A. A., Morgan T. M., Mathews C. A., Pauls D. L., Rasin M. R., Gunel M. et al.: Sequence variants in SLITRK1 are associated with Tourette's syndrome. Science 2005, 310, 317-320.

2. Ambros V., Bartel B., Bartel D. P., Burge C., Carrington J. C., Chen X. et al.: A uniform system for microRNA annotation. RNA 2003, 9, 277-279.

3. Barrey E., Bonnamy B., Barrey E. J., Mata X., Chaffaux S., Guerin G.: Muscular microRNA expressions in healthy and myopathic horses suffering from polysaccharide storage myopathy or recurrent exertional rhabdomyolysis. Equine Vet. J. 2010, 42, 303-310.

4. Bartel D. P.: MicroRNAs: Genomics, biogenesis, mechanism, and function. Cell 2004, 116, 281-297.

5. Basyuk E., Suavet F., Doglio A., Bordonne R., Bertrand E.: Human let-7 stemloop precursors harbor features of RNase III cleavage products. Nucleic Acids Res. 2003, 31, 6593-6597.

6. Bhaskaran M., Mohan M.: MicroRNAs History, Biogenesis, and Their Evolving Role in Animal Development and Disease. Vet. Pathol. Online 2014, 51, 759-774 .

7.Boggs R. M., Wright Z. M., Stickney M. J., Porter W. W., Murphy K. E.: MicroRNA expression in canine mammary cancer. Mamm. Genome 2008, 19, 561-569. doi: 10.1007/s00335-008-9128-7.

8. Brennecke J., StarkA., Russell R. B., Cohen S. M.: Principles of microRNA-target recognition. PLoS Biol 2005, 3, e85. doi: 10.1371/journal.pbio.0030085.

9. Buechli M. E., Lamarre J., Koch T. G.: MicroRNA-140 expression during chondrogenic differentiation of equine cord blood-derived mesenchymal stromal cells. Stem Cells Dev. 2013, 22, 1288-1296.

10. Cai X., Lu S., Zhang Z., Gonzalez C. M., Damania B., Cullen B. R.: Kaposi's sarcoma-associated herpesvirus expresses an array of viral microRNAs in latently infected cells. Proc. Natl. Acad. Sci. 2005, 102, 5570

11. Calin G. A, Croce C. M.: MicroRNA signatures in human cancers. Nat. Rev. 2006, 6, 857-866.

12. Cameron A., da Silveira J. C., Bouma G., Bruemmer J. E.: Evaluation of Exosomes Containing miRNA as an Indicator of Pregnancy Status in the Mare. J. Equine Vet. Sci. 2011, 31, 314-315.

13. Carrington J. C., Ambros V.: Role of microRNAs in plant and animal development. Science 2003, 301, 336-338.

14. Castro F. O., Sharbati S., Rodríguez-Alvarez L. L, Cox J. F., Hultschig C., Einspanier R.: MicroRNA expression profiling of elongated cloned and in vitro-fertilized bovine embryos. Theriogenology 2010, 73, 71-85.

15. Chen J. F., Mandel E. M., Thomson J. M., Wu Q., Callis T. E., Hammond S. M., Conlon F. L., Wang D. Z.: The role of microRNA-1 and microRNA-133 in skeletal muscle proliferation and differentiation. Nat. Genet. 2006, 38, 228-233.

16. Donadeu F. X., Schauer S. N.: Differential miRNA expression between equine ovulatory and anovulatory follicles. Domest. Anim. Endocrinol. 2013, 45, 122-125. 
17. Dunn W., Du Raine G., Reddi A. H.: Profiling microRNA expression in bovine articular cartilage and implications for mechanotransduction. Arthritis Rheum 2009, 60, 2333-2339

18. Fenger J. M., Volinia S., Jalkanen A., Gulcin Ozer H., Sarver A. L., Subramanian S., Breen M., Modiano J., London Ch., Kisseberth W.: 10.1158/1538-7445. AM2012-184 Cancer Res. 2012, 72 (8 Supplement), 184

19. Fleischhacker S. N., Bauersachs S., Wehner A., Hartmann K., Weber K. Differential expression of circulating microRNAs in diabetic and healthy lean cats. Vet. J. 2013, 197, 688-693.

20. Govindaraju A., Uzun A., Robertson L., Atli M. O., Kaya A., Topper E., Crate E. A., Padbury J., Perkins A., Memili E.: Dynamics of microRNAs in bull spermatozoa. Reprod. Biol. Endocrinol. 2012, 14, 10, 82

21. Grey F., Antoniewicz A., Allen E., Saugstad J., McShea A., Carrington J. C. Nelson J.: Identification and characterization of human cytomegalovirus-encoded microRNAs. J. Virol. 2005, 79, 12095-12099.

22. Griffiths-Jones S.: The microRNA Registry. Nucleic Acids Res 2004, 32 (Database issue): D109-11. doi:10.1093/nar/gkh023. PMC 308757. PMID 14681370 .

23. Grundhoff A., Sullivan C. S., Ganem D.: A combined computational and microarray-based approach identifies novel microRNAs encoded by human gamma-herpesviruses. RNA 2006, 12, 733-250.

24. Guo X. K., Zhang Q., Gao L., Li N., Chen X. X., Feng W. H.: Increasing expression of micro RNA 181 inhibits porcine reproductive and respiratory syndrome virus replication and has implications for controlling virus infection. J. Virol. 2013, 87, 1159-1171.

25. He L., Thomson J. M., Hemann M. T., Hernando-Monge E., Mu D., Goodson S. Powers S., Cordon-Cardo C., Lowe S. W., Hannon G. J., Hammond S. M. A microRNA polycistron as a potential human oncogene. Nature 2005, 435, 828-833.

26. Hulanicka M., Garncarz M., Parzeniecka-Jaworska M., Jank M.: Plasma miRNAs as potential biomarkers of chronic degenerative valvular disease in Dachshunds. BMC Vet. Res. 2014, 10, 205 . doi:10.1186/s12917-014-0205-8.

27. Hwang H. W., Mendell J. T.: MicroRNAs in cell proliferation, cell death, and tumorigenesis. Br. J. Cancer 2006, 94, 776-780.

28. Ichii O., Otsuka S., Ohta H., Yabuki A., Horino T., Kon Y.: MicroRNA expression profiling of cat and dog kidneys. Res. Vet. Sci. 2014, 96, 299-303.

29. Ji Z., Wang G., Xie Z., Wang J., Zhang C., Dong F., Chen C.: Identification of novel and differentially expressed MicroRNAs of dairy goat mammary gland tissues using solexa sequencing and bioinformatics., PLoS One 2012,;7 (11): e49463. doi: 10.1371/journal.pone.0049463.

30. Johnston R. J., Hobert O.: A microRNA controlling left/right neuronal asymmetry in Caenorhabditis elegans. Nature 2003, 426, 845-849.

31. Kim J., Krichevsky A., Grad Y., Hayes G. D., Kosik K. S., Church G. M. Ruvkun $G$.: Identification of many microRNAs that copurify with polyribosomes in mammalian neurons. Proc. Natl. Acad. Sci. 2004, 101, 360-365.

32. Kloosterman W. P., Wienholds E., de Bruijn E., Kauppinen S., Plasterk R. H.: In situ detection of miRNAs in animal embryos using LNA-modified oligonucleotide probe. Nat. Methods 2006, 3, 27-29.

33. Lee R. C., Feinbaum R. L., Ambros V.: The C. elegans heterochronic gene lin-4 encodes small RNAs with antisense complementarity to lin-14. Cell 1993, 75, 843-8542.

34. Lee Y., Ahn C., Han J., Choi H., Kim J., Yim J., Lee J., Provost P., Radmark O., Kim S., Kim V. N.: The nuclear RNase III Drosha initiates microRNA processing. Nature 2003, 425, 415-419.

35. Lee Y., Jeon K., Lee J. T., Kim S., Kim V. N.: MicroRNA maturation: stepwise processing and subcellular localization. Embo J. 2002, 21, 4663-4670.

36. Li Z., Liu H., Jin X., Lo L., Liu J.: Expression profiles of microRNAs from lactating and non-lactating bovine mammary glands and identification of miRNA related to lactation. BMC Genomics 2012, 13, 731, doi: 10.1186/1471-216413-731.

37. Li Z., Wang H., Chen L., Wang L., Liu X., Ru C., Song A.: Identification and characterization of novel and differentially expressed microRNAs in periphera blood from healthy and mastitis Holstein cattle by deep sequencing. Anim. genet. $2014,45,20-27$

38. Liu J., Valencia-Sanchez M. A., Hannon G. J., Parker R.: MicroRNA-dependent localization of targeted mRNAs to mammalian P-bodies. Nat. Cell. Biol. 2005, 7, 719-723.

39. Lund E., Guttinger S., Calado A., Dahlberg J. E., Kutay U.: Nuclear export of microRNA precursors. Science 2004, 303, 95-98.

40. Martinez J., Patkaniowska A., Urlaub H., Lührmann R., Tuschl T.: Single stranded antisense siRNA guide target RNA cleavage in RNAi. Cell 2002, 110 , 563-574

41. Naguibneva M., Ameyar-Zazoua A., Polesskaya S., Ait-Si-Ali R., Groisman M. Souidi S., Cuvellier A.: The microRNA miR-181 targets the homeobox protein Hox-A11 during mammalian myoblast differentiation. Nat. Cell Biol. 2006, 8, 278-284

42. Olsen P. H., Ambros V.: The lin-4 regulatory RNA controls developmental timing in Caenorhabditis elegans by blocking LIN-14 protein synthesis after the initiation of translation. Dev. Biol. 1999, 216, 671-680.
43. Plasterk R. H. A.: Micro RNAs in Animal Development. Cell 2006, 124, 877-881 doi 10.1016/j.cell.2006.02.030.

44. Podolska A., Anthon C., Bak M., et al.: Profiling microRNAs in lung tissue from pigs infected with Actinobacillus pleuropneumoniae. BMC Genomics 2012, 13, 459.

45. Potenza N., Papa U., Mosca N., Zerbini F., Nobile V., Russo A.: Human microRNAhsa-miR-125a $5 \mathrm{p}$ interferes with expression of hepatitis B virus surface antigen. Nucleic Acids Res. 2011, 39, 5157-5163.

46. Redshaw N., Wilkes T., Whale A., Cowen S., Huggett J., Foy C. A.: A comparison of miRNA isolation and RT-qPCR technologies and their effects on quantification accuracy and repeatability. Biotechniques 2013, 54, 155-164.

47. Rodriguez A., Griffiths-Jones S., Ashurst J. L., Bradley A.: Identification of mammalian microRNA host genes and transcription units. Genome Res. 2004, 14, 1902-1910.

48. Samols M. A., Hu J., Skalsky R. L., Renne R.: Cloning and identification of a microRNA cluster within the latency-associated region of Kaposi's sarcomaassociated herpesvirus. J. Virol. 2005, 79, 9301.

49. Schauer S. N., Sontakke S. D., Watson E. D., Esteves C. L., Donadeu F. X. Involvement of miRNAs in equine follicle development. Reproduction 2013, 146, 273-282. doi: 10.1530/REP-13-0107.

50. Sen G. L., Blau H. M.: Argonaute 2/RISC resides in sites of mammalian mRNA decay known as cytoplasmic bodies. Nat. Cell. Biol. 2005, 7, 633-636.

51. Siengdee P., Trakooljul N., Murani E., Brand B., Schwerin M., Wimmers K., Ponsuksili S.: Pre-and post-natal muscle microRNA expression profiles of two pig breeds differing in muscularity. Gene 2015, 561, 190-198.

52. Silveira J. C. da, Veeramachaneni D. N., Winger Q. A., Carnevale E. M., Bouma $G$. $J$ : Cell-secreted vesicles in equine ovarian follicular fluid contain miRNAs and proteins: a possible new form of cell communication within the ovarian follicle. Biol. Reprod. 2012, 19, 86,71. doi: 10.1095/biolreprod.111.093252.

53. Steudemann C., Bauersachs S., Weber K., Wess G.: Detection and comparison of microRNA expression in the serum of Doberman Pinschers with dilated cardiomyopathy and healthy controls. BMC Vet. Res. 2013, 9, 12, doi: 10.1186/17466148-9-12.

54. Tang Q., Wu Y. Q., Chen D. S., Zhou Q., Chen H. C., Liu Z. F.: Bovine herpesvirus 5 encodes a unique pattern of microRNAs compared with Bovine herpesvirus 1. J. Gen. Virol. 2014, 95, 671-678. doi: 10.1099/vir.0.061093-0.

55. Tesfaye D., Worku D., Rings F., Phatsara C., Tholen E., Schellander K. Hoelker M.: Identification and expression profiling of microRNAs during bovine oocyte maturation using heterologous approach. Mol. Reprod. Dev. 2009, 76, 665-677. doi: 10.1002/mrd.21005

56. Tian F., Luo J., Zhang H., Chang S., Song J.: MiRNA expression signatures induced by Marek's disease virus infection in chickens. Genomics 2012, 99 , $152-159$

57. Tuddenham L., Wheeler G., Ntounia-Fousara S., Waters J., Hajihosseini M. K. Clark I., Dalma T.: The cartilage specific microRNA-140 targets histone deacetylase 4 in mouse cells FEBS Lett. 2006, 580, 4214-4217.

58. Uhl W. E., Suter S., Antes T., Tompkins S. M.: MiRNA Profiles of Canine Lymphoid Cell Line. FASEB J. 2009, April 23 (Meeting Abstract Supplement), 361.3

59. Uhl W. E. Suter $S$., Krimer P., Schliekelman P., Tompkins $S$. M. Suter S. Identification of altered MicroRNA expression in canine lymphoid cell lines and cases of B- and T-Cell lymphomas. Genes Chromosomes Cancer 2011, 950-967. doi: 10.1002/gcc.20917.

60. Uhl W. E., Suter S., Krimer P., Tompkins S. M., Lester C. H., Suter S.: MiRNA Profiles of Spontaneous Canine Lymphoid Tumors, FASEB J. 2010, April, 24 (Meeting Abstract Supplement) 1027.8.

61. Weber K., Rostert N., Bauersachs S., Wess G.: Serum microRNA profiles in cats with hypertrophic cardiomyopathy. Mol. Cell. Biochem. 2015, 402, 171-180.

62. Wicik Z., Gajewska M., Majewska A., Walkiewicz D., Osińska E., Motyl T. Characterization of microRNA profile in mammary tissue of dairy and beef breed heifers. J. Anim. Breed Genet. 2016, 133, 31-42.

63. Wienholds E., Plasterk R. H.: MicroRNA function in animal development. FEBS lett. 2005, 579, 5911-5922.

64. Vinall R. L., Kent M. S., deVere White R.: White Expression of microRNAs in urinary bladder samples obtained from dogs with grossly normal bladders, inflammatory bladder disease, or transitional cell carcinoma. Am. J. of Vet. Res. 2012, 73, 1626-1633.

65. Yi R., Qin Y., Macara I. G., Cullen B. R.: Exportin-5 mediates the nuclear export of pre-microRNAs and short hairpin RNAs. Genes Dev. 2003, 17, 3011-3016.

66. Yin J. Q., Zhao R. C., Morris K. V.: Profiling microRNA expression with microarrays. Trends Biotechnol. 2008, 26, 70-76.

67. Zeng Y., Cullen B. R.: Sequence requirements for micro RNA processing and function in human cells. RNA 2003, 9, 112-123.

68. Zhao P., Zhao L., Zhang T., Wang H., Qin C., Yang S., Xia X.: Changes in microRNA expression induced by rabies virus infection in mouse brains. Microb. Pathog. 2012, 52, 47-54.

Corresponding author: prof. dr hab. Piotr Ostaszewski, Nowoursynowska 159, 02-776 Warsaw, Poland; e-mail: piotr_ostaszewski@sggw.pl 